\title{
The Application of Computational Geometry Algorithms in Discriminating Direct Injection-Production Connections
}

\author{
Zhang Jiqun $^{1, \mathrm{a}}$, Hu Chungjin ${ }^{1, \mathrm{~b}}$, Deng Baorong ${ }^{2, \mathrm{c}}$, Li Xinhao ${ }^{2}, \mathrm{He}^{2}$ Dongmei $^{3}$, Li \\ Hua $^{2}$, Chang Junhua ${ }^{2}$ \\ ${ }^{1}$ University of Science and Technology Beijing, Beijing, 100083, China \\ ${ }^{2}$ Research Institute of Petroleum Exploration \& Development, Beijing, 100083, China \\ ${ }^{3}$ Petrochina Planning and Engineering Institute, Beijing, 100083, China \\ aemail: zjqwxs@sina.com, bemail: huchungjun@sina.com, cemail: dbr@petrochina.com.cn
}

\begin{abstract}
Keywords: Direct Connection, Least Resistance Path, Injection-Production Connection, Computational Geometry, Sand Body, Fault
\end{abstract}

\begin{abstract}
It is necessary to analyze direct connections between injection wells and production wells for discovering superior water channels, researching water flooding effectiveness and flow directions in a single well group. A new method of discriminating the direct connections by applying computational geometry algorithms and percolation theory was proposed. This method uses static and dynamic, monitoring and stimulation data and considers various factors such as patterns and distributions of sand bodies and faults, production performance and perforation sealing state. Experiment results in dozens of oilfield blocks demonstrate that the method has advantages of high accuracy and high calculation speed.
\end{abstract}

\section{Introduction}

Most oilfields in China are continental deposit and have many sandstone oil layers, causing severe heterogeneity among layers. In later stage of high water cut, development contradictions in a layer and among layers become gradually prominent and the phenomenon of water flooding ineffective circulations occurs. Low effectiveness of injecting water brings about difficulties in stabilizing oil production and controlling water production, rapid ascent of water cut in production wells and low water flooding producing degree, directly influencing water flooding development effect and economic benefits. As a result, it is necessary to analyze direct injection-production connections for discovering superior water channels, researching water flooding effectiveness and flow directions in a single well group. Thereby foundations for injection-production layers adjustment, well pattern improvement, water shut-off and profile control, and eventually oil recovery enhancement are provided.

Currently, there are mainly two types of ways to research injection-production connections. One type is testing methods among wells, including geochemistry, pressure testing, isotope tracer, interference well testing, cable logging and so on. The other type is methods of applying mathematical techniques to analyze static and dynamic data, for example, decision tree [1], grey correlation [2], data inversion [3], time series analysis [4], multiple linear regression analysis [5] and filtering algorithm [6]. The former type is quantitative analysis having accurate and reliable results. Normally oilfields however only use them in key well groups because of their high costs and long periods. The latter type is analytic methods with the characteristics of low costs and short periods. But limit data types and factors, generally only well production data and a few static data such as permeability, effective thickness and porosity, are considered in most algorithms, causing inaccurate analysis results.

A new method of layer by layer discriminating direct connections between injection wells and production wells by applying computational geometry algorithms and percolation theory was proposed. This method uses dozens of types of data, including static and dynamic, monitoring and stimulation data, and considers various factors. 


\section{Injection-production Connection Discrimination Method}

\section{Basic Data}

Six types of data are needed to discriminate injection-production connections: 1 , the type of basic reservoir data, such as crude oil viscosity; 2, the type of reservoir property data of wells in each layer, such as permeability, effective thickness, sandstone thickness, top depth and bottom depth of sandstone; 3, the type of injection and production data of wells, such as monthly production data in history and monthly injection data in history; 4, the type of stimulation data, such as perforation, perforation adding, plugging and fracturing; 5, the type of production logging data, such as tracer; 6 , the type of boundary data, such as sand body boundaries, oil sand body boundaries, non-flow boundaries of oil and water.

\section{Discrimination Rules}

The existence of tracer data between two wells should be examined at first to discriminate direct injection-production connections. If the data exist and show that isotopes injected in an injection well are found in a production well, we assume the production well is connected to the injection well. However because of the scarcity of tracer data among wells, in most conditions, direct injection-production connections discrimination should consider many other factors. The direct connections should satisfy following five rules at the same time.

Discrimination Rule 1: An injection well and a production well must be in the same sand body.

Only if an injection well and a production well are in the same sand body, oil and water are likely to flow between them, which means connections cannot exist in wells not in the same sand body, as Fig. 1(a) shown.

Discrimination Rule 2: An injection well and a production well must be open in the same layer at the same time.

Only if a well is injecting while a well is producing at the same layer, they are likely to be connected. According to layer property data, injection and production history data and stimulation data such as such as perforation, perforation adding and plugging, whether an injection well was injecting in a layer at some date and whether a production well was producing in a layer at some layer can be calculated. If an injection well was injecting in a layer while a production well was not producing in the same layer, they were not connected in the layer at that time, as Fig. 1(b) shown.

Discrimination Rule 3: A connected path must exist in an injection well and a production well

If an injection well and a production well are in the same sand body, a connected path where oil and water approximately flow between them needs to be found. If obstacles like fault or mudstone in sand bodies, whether they block oil and water to flow between the wells should be judged, as Fig. 1(c) and (d) shown.

Discrimination Rule 4: There must be no interference wells between an injection well and a production well

Interference wells are open wells in some certain range between an injection well and a production well. The existence of interference wells greatly reduces or even eliminates the ability of oil and water to flow among wells. Fig. 1(e) shows that Well Inj 2 exits between well Inj ${ }_{1}$ and Oil ${ }_{1}$. We assume that $\mathrm{Oil}_{1}$ is indirectly connected to $\mathrm{Inj}_{1}$.

Discrimination Rule 5: Pressure conduction must be reasonable from an injection well to a production well

Water flows only in one direction in any locations besides its injected location. As a result, when two connected paths of a pair of injection wells and production wells are crossed as Fig. 1(f) shown, $\mathrm{R}_{1}$ and $\mathrm{R}_{2}$ cannot be valid at the same time. Water from injection wells flows toward the direction to production wells which has lower resistance.

\section{The Injection-production Connection Discrimination Algorithm and Its Flow}

\section{Algorithms}

Among the five discrimination rules above, rule 2, whether an injection well and a production well are open in the same layer at the same time, can be directly judged from injection and 
production history data and stimulation data such as such as perforation, perforation adding and plugging. The other four rules need to be judged by following computational geometry algorithms.

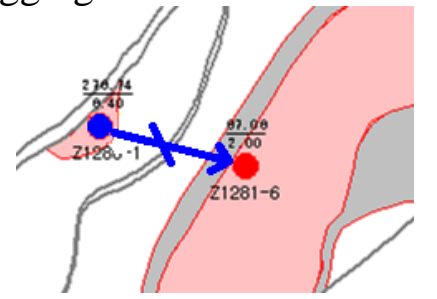

(a) Sand Body Distributions

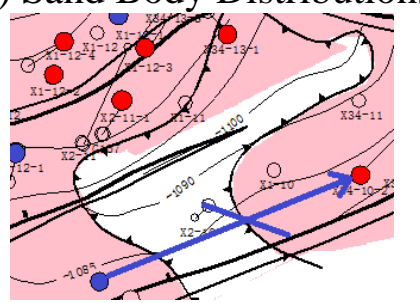

(d) Mudstone Zone Blocking

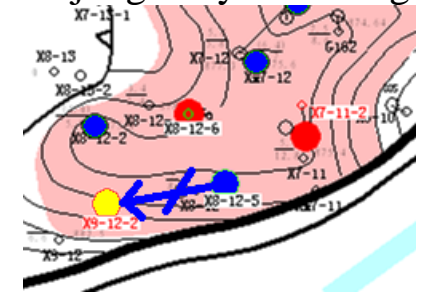

(b) Production Conditions

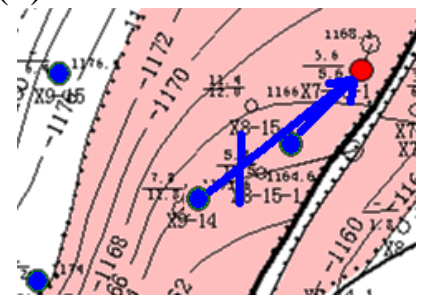

(e) Direct Connections

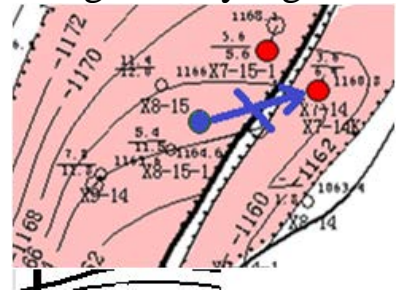

(c) Fault Blocking

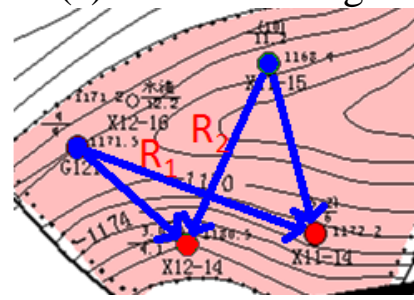

(f) Pressure Conduction

Fig.1 Injection-production Connection Discrimination Rules

(1) The algorithm to judge whether an injection well and a production well are in the same sand body

In discrimination rule 1 , whether an injection well and a production well are in the same sand body should be judged. To do this, we apply the "Ray Method" of computational geometry to calculate which sand body each well locates at. The description of algorithm is as follows.

We consider the boundary of a sand body as a polygon and the well location coordinate as a point, and then judge whether the point is in the polygon, as Fig. 2shown.

"Ray Method" Algorithm:

Input parameters: coordinate of a well, the inner and outer boundary of sand body

Output parameters: the sand body where the well locates

1) Set a ray from the well point horizontally (or vertically), called scan line.

2) Calculate the number of intersection points of the scan line and the inner and outer boundary of sand body.

3) When they intersect at a vertex, if the adjacent vertices are in the same side of the scan line, the number adds one.

4) When the scan line coincides with an edge, the number is one.

5) When the number of intersection points is odd, the point is in the polygon. Else, the point is out of the polygon.

In Fig. 2, the ray from production well Oil ${ }_{1}$ has three intersection points with boundary of sand body 1 , and has two with sand body 2, so $\mathrm{Oil}_{1}$ is in sand body 1 rather sand body 2. Similarly, the ray from injection well $\operatorname{Inj}_{1}$ has one intersection points with boundary of sand body 1 , so $\operatorname{Inj}_{1}$ in sand body 1 . The ray from injection well $\operatorname{Inj}_{2}$ has one intersection points with boundary of sand body 2, so $\mathrm{Inj}_{2}$ in sand body 2 . Therefore, $\mathrm{Oil}_{1}$ and $\mathrm{Inj}_{1}$ are in the same sand body and possibly direct connected. Oil 1 and Inj $_{2}$ are not in the same sand body and impossibly direct connected.

(2) Judgment of interference wells between an injection well and a production well

To judge existence of interference wells between an injection well and a production well equals to whether they are directly connected or indirectly connected. Interference wells greatly reduce or even eliminate the ability of oil and water to flow among wells. We neglect this extremely weak flow connection. Only wells are injecting or producing can be considered as interference wells, while close or imperforated wells cannot be.

As Fig. 3 demonstrated, a polygon should be constructed according to the distance from $\mathrm{Oil}_{0}$ to Inj $_{0}$ at first to judge whether they are directly connected. If there is an injection well in the green region of Fig. 3(a), then $\mathrm{Oil}_{0}$ is not directly connected toInj ${ }_{0}$. Inj 7 is in the green region, so Oil ${ }_{0}$ is not directly connected to Inj $_{0}$. Similarly, if there is a production well in the green region of Fig. 3(b), thenOil $l_{0}$ is not directly connected toInj $0_{0} \mathrm{Oil}_{7}$ is in the green region, so $\mathrm{Oil}_{0}$ is not directly 
connected toInj ${ }_{0}$. The "Ray Method" above is used to judge whether there are wells in the green polygon.

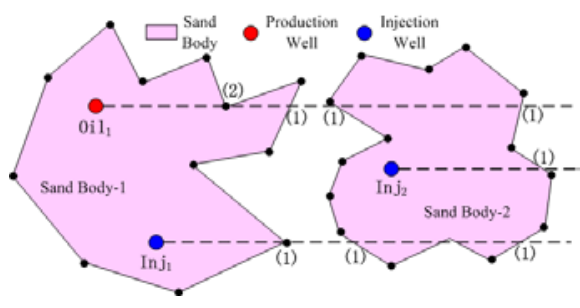

Fig.2 Points in Polygons

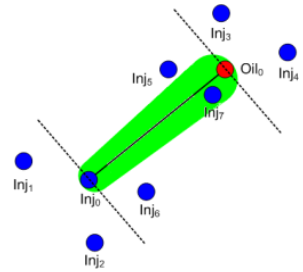

(a) Interference Injection Well

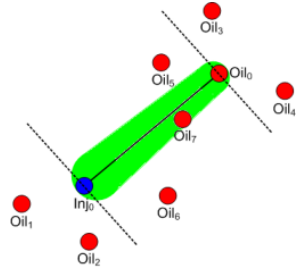

(b) Interference Production Well Fig.3 Interference Well Judgment

(3) The algorithm to calculate the least resistance path from an injection well to a production well

This algorithm realizes judgments of discrimination rule 3 and 5.The injected water must flow the least resistance path to production wells, so we need to calculate the least resistance path. We apply the "Point Robot Shortest Path Based on Visibility Graph Algorithm". The algorithm is illustrated by the example shown in Fig. 4 as follows.

Procedure 1: To reservoir blocks with strong heterogeneity, the fields of permeability and thickness should be established at first. Data from geological modeling can be used. We take the graph of permeability field as example, as Fig. 5 shown.

Procedure 2: Construct visibility graph based field data.

The center points of all grids in field data are seen as the vertices of visibility graph, denoted by union A. Considering the inner and outer boundary of sand body (denoted by union $\mathrm{C}$ ) and the boundary of non-flow fault (denoted by union F), visibility graph is constructed by the following algorithm.

Algorithm name: VisibilityGraph [7]

Input parameters: union $\mathrm{A}$, union $\mathrm{C}$ and union $\mathrm{F}$ defined above

Output parameters: visibility graphGvis(A)

1) Initialize $G \operatorname{vis}(A)=(V, E)$, set union A including all the vertices, $E=\varnothing$,

2) for (each vertex $v \in V$ ),

3) do W $\leftarrow$ VisibilityVertices(v, A, C, F),

4) for each vertex $w \in W$, add $\operatorname{arc}(v, w)$ to $E$,

5) returnGvis(A).

Algorithm name: VisibilityVertices

Input parameters: union $\mathrm{C}$ and union $\mathrm{F}$ defined above, union $\mathrm{A}$ which includes all vertices in the visibility graph, a point $\mathrm{v}$ without any obstacles.

Output parameters: the union $\mathrm{W}$ of all vertices in the visibility graph that can visualize point $\mathrm{V}$

1) According to the clockwise angle between half-line across $v$ and each vertex and the $X$ axis, all vertices are sequenced from small angles to large angles. If two vertices have the equal angle, the one closer to $\mathrm{V}$ is ordered in front of the other one. The list of order vertices: $\mathrm{w}_{1} \ldots \mathrm{w}_{\mathrm{n}}$.

2) Let $\rho$ is the half-line starting from $v$ and parallel with the $X$ axis. Find all obstacle edges that intersect $\rho$ at points besides the vertices. Store the edges into a binary search tree in the order of intersection with $\rho$.

3) $\mathrm{W} \leftarrow 0$.

4) for $\mathrm{i} \leftarrow 0$ to $n$.

5) do if $w_{i}$ is visible, then add $w_{i}$ to $W$.

6) In the obstacle edges connected with $w_{i}$, find all edges lie in clockwise side of half-line $\mathrm{Vw}_{\mathrm{i}}$ and add them into $\mathrm{T}$.

7) In the obstacle edges connected with $w_{i}$, find all edges lie in counterclockwise side of half-line $\mathrm{vw}_{\mathrm{i}}$ and delete them from $\mathrm{T}$.

8) return $W$.

Fig. 6 shows unweighted visibility graph constructed from Fig. 5. 


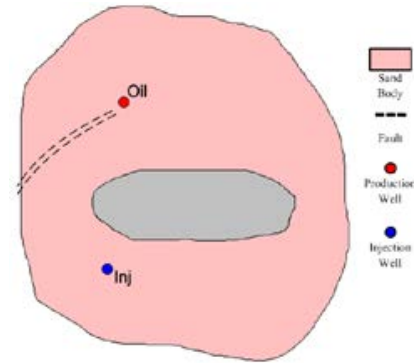

Fig. 4 Sand Body

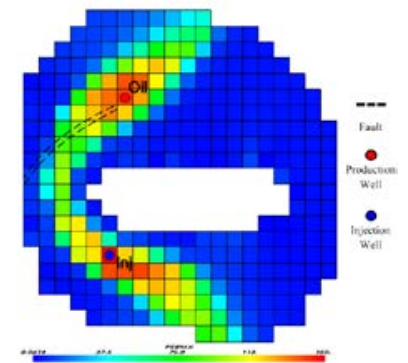

Fig. 5 Permeability

Containing Inj and Oil Field of the Sand Body
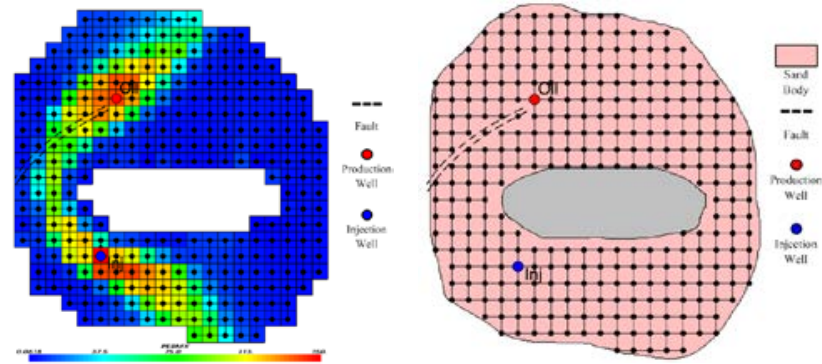

Fig.6 Unweighted Visibility

Graph of Sand Body

Procedure 3: Calculate the weight of each line in visibility graph, called quasi-resistance. The value of quasi-resistance is related to data of permeability, thickness, pressure and so on. Fig. 7 shows the flow of oil and water in two grids. $R_{i, j \rightarrow i+1, j}$, the of quasi-resistance from grid (i,j) to grid $(\mathrm{i}+1, \mathrm{j})$ is calculated by Eq. 1

$$
R_{i, j \rightarrow i+1, j}=\frac{1}{\frac{K r w}{\mu_{w}}+\frac{K r o}{\mu_{o}}} \times \frac{\frac{1}{2} \times\left(\frac{D x_{i, j}}{K_{i, j}}+\frac{D x_{i+1, j}}{K_{i+1, j}}\right)}{\left(\frac{H_{i, j}+H_{i+1, j}}{2}\right) \times\left(\frac{D y_{i, j}+D y_{i+1, j}}{2}\right)} \times \frac{1}{\left(P_{i, j}-P_{i+1, j}\right)}
$$

$\mathrm{DX}_{\mathrm{i}, \mathrm{j}}$ : the $\mathrm{X}$ direction width of grid in row $\mathrm{i}$, column $\mathrm{j}$

$\mathrm{DY}_{\mathrm{i}, \mathrm{j}}$ : the $\mathrm{Y}$ direction width of grid in row $\mathrm{i}$, column $\mathrm{j}$

$\mathrm{K}_{\mathrm{i}, \mathrm{j}}$ : the permeability of grid in row $\mathrm{i}$, column $\mathrm{j}$

$\mathrm{H}_{\mathrm{i}, \mathrm{j}}$ : the thickness of grid in row $\mathrm{i}$, column $\mathrm{j}$

$\mathrm{P}_{\mathrm{i}, \mathrm{j}}$ : the pressure of grid in row $\mathrm{i}$, column $\mathrm{j}$

Krw: water relative permeability

Kro: oil relative permeability

According to equation (1), local weighted visibility graph is obtained as Fig. 8 after calculating quasi-resistance values of all line.

Procedure 4: Based on the weighted visibility graph, apply the Dijkstra Algorithm [8] to calculate the least resistance path from injection well Inj to production well Oil.

From left to right and from top to bottom, all vertices in the visibility graph is marked as $1,2 \ldots$ $\mathrm{n}$. The description of Dijkstra Algorithm with n vertices is as follows.

Algorithm name: Dijkstra

Input parameters: visibility graph Gvis(A)

Output parameters: the least resistance paths between all pairs of vertices.

1) Set $\bar{S}=\{2,3, \cdots, n\}, \pi(1)=0, \pi(i)=\left\{\begin{array}{cc}l_{1 i} & i \in \tau_{1}^{+} \\ \infty & \text { other }\end{array}, \bar{S}\right.$ is the union of all nodes whose least resistance paths are not found

2) Find $j$ satisfying $\pi(j)=\min _{i \in \bar{S}} \pi(i)$, in $\bar{S}, \bar{S} \leftarrow \bar{S}-\{j\}$, if $\bar{S}=\emptyset$, end, or go to 3)

3) For all $i \in \bar{S} \cap \tau_{1}^{+}$, set $\pi(i) \leftarrow \min \left(\pi(i), \pi(j)+l_{1 i}\right)$, go to 2$)$

Applying the Dijkstra Algorithm, the least resistance path from injection well Inj to production well Oil is calculated, as Fig. 9 shown.

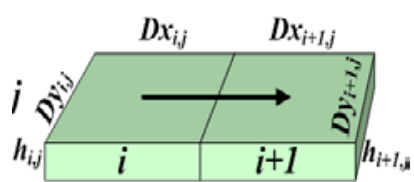

Fig.7 Flow of Oil and Water in Two Grids

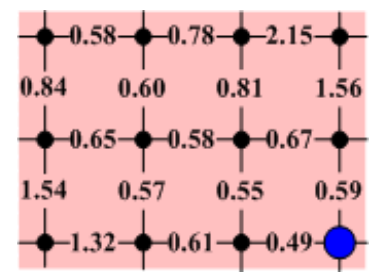

Fig.8 Local Weighted Visibility Graph of Sand Body
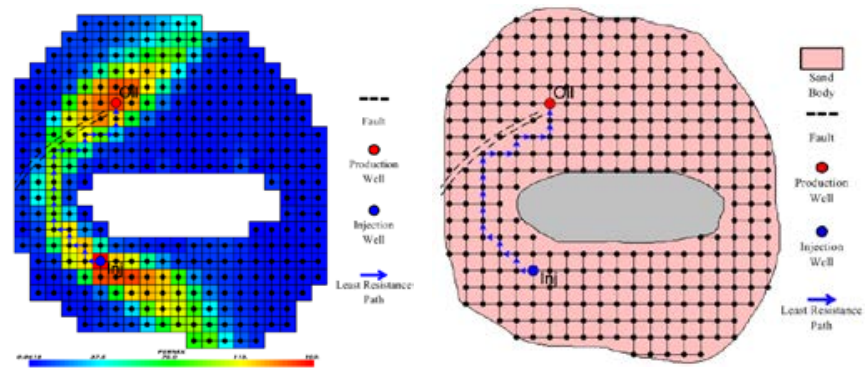

Fig.9 The Least Resistance Path from Injection Well Inj to Production Well Oil

When two paths in discrimination rule 5 intersect, the two wells which have smaller flow resistance path are connected. In Fig. $1(f)$, if $R_{1}>R_{2}$, the two wells linked by $R_{2}$ are directly 
connected.

The Flow of Injection-production Connection Discrimination Algorithm

Without tracer data, according algorithms we proposed, the algorithm flow chart to discriminate direct injection-production connections in some layer at some date in a reservoir block is given in Fig. 10.

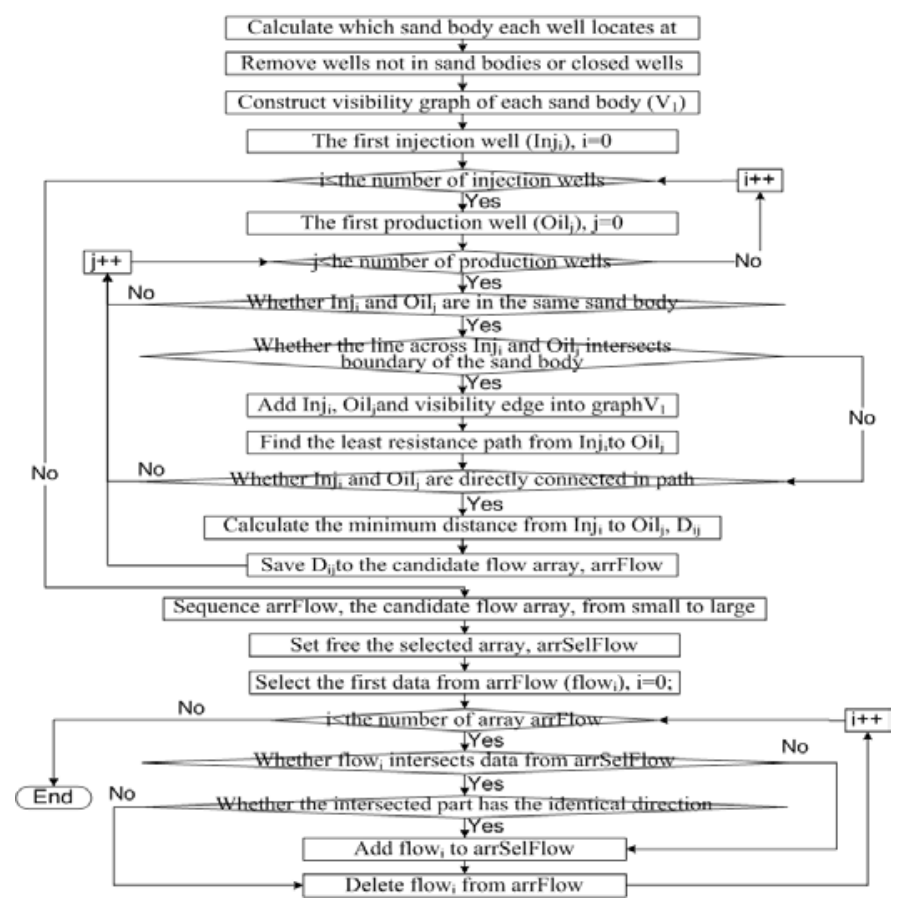

Fig.10 The Algorithm Flow of Direct Injection-production Connection Discrimination in a Layer at Some Date

\section{Application Example}

Taking a certain oilfield block with complex faults in east China as an example, the block has 42 layers and 237 wells. Fig. 11 shows direct connections between injection wells and production wells in four major layers discriminated by our method. According to direct injection-production connections in layers, the number of connected wells in sand bodies and water flooding control degree can be obtained. The numbers of one directional, two directional, three directional and multiple directional injection-production connections in each sand body in the four major layers are summarized in table 1.
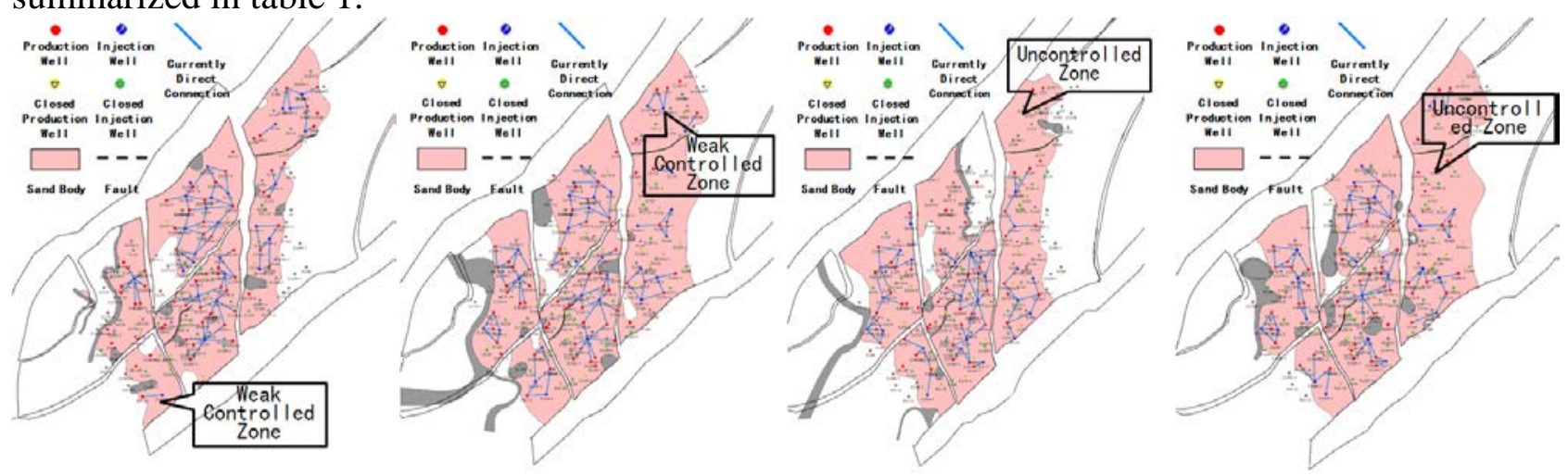

Fig.11 Direct Injection-Production Connections in a Certain Block with Faults

After direct injection-production connection analysis layer by layer, sand bodies thought to be controlled may be not in control or in weak control. The final recovery factor of water flooding and enhanced oil recovery can be increased by improving direct injection-production connection in well patterns of secondary development and enhanced oil recovery.

The software programmed by our algorithms has been used in dozens of oilfield blocks. Their results demonstrate that the method can rapidly discriminate direct injection-production connections 
in water flooding sandstone reservoirs and have already achieved the unity of accuracy and efficiency.

Table 1 Numbers of Direct Injection-Production Connections in Each Sand Body

\begin{tabular}{|c|c|c|c|c|c|c|c|}
\hline \multirow{2}{*}{$\begin{array}{l}\text { Layer } \\
\text { Name }\end{array}$} & \multirow{2}{*}{$\begin{array}{c}\text { Sand } \\
\text { Body } \\
\text { Number }\end{array}$} & \multicolumn{5}{|c|}{ Numbers of Injection-Production Connections } & \multirow{2}{*}{$\begin{array}{c}\text { Connection } \\
\text { Ratio (\%) }\end{array}$} \\
\hline & & None & $\begin{array}{c}\text { One } \\
\text { Directional }\end{array}$ & $\begin{array}{c}\text { Two } \\
\text { Directional }\end{array}$ & $\begin{array}{c}\text { Three } \\
\text { Directional }\end{array}$ & $\begin{array}{c}\text { Multiple } \\
\text { Directional }\end{array}$ & \\
\hline \multirow{7}{*}{ 2c2 } & 1 & 2 & 3 & 0 & 2 & 0 & 71.43 \\
\hline & 2 & 1 & 4 & 2 & 1 & 0 & 87.50 \\
\hline & 3 & 1 & 2 & 2 & 6 & 2 & 92.31 \\
\hline & 4 & 2 & 11 & 6 & 1 & 0 & 90.00 \\
\hline & 5 & 4 & 7 & 0 & 0 & 0 & 63.64 \\
\hline & 6 & 2 & 1 & 0 & 0 & 0 & 33.33 \\
\hline & 7 & 0 & 0 & 0 & 0 & 0 & 0.00 \\
\hline \multirow{7}{*}{$3 c 2$} & 1 & 2 & 2 & 2 & 0 & 0 & 66.67 \\
\hline & 2 & 2 & 4 & 3 & 2 & 0 & 81.82 \\
\hline & 3 & 0 & 0 & 0 & 0 & 0 & 0.00 \\
\hline & 4 & 1 & 1 & 3 & 3 & 1 & 88.89 \\
\hline & 5 & 3 & 9 & 6 & 1 & 0 & 84.21 \\
\hline & 6 & 1 & 4 & 7 & 1 & 0 & 92.31 \\
\hline & 7 & 0 & 0 & 2 & 1 & 0 & 100.00 \\
\hline \multirow{7}{*}{ 3c3 } & 1 & 3 & 1 & 2 & 0 & 0 & 50.00 \\
\hline & 2 & 4 & 5 & 3 & 1 & 0 & 69.23 \\
\hline & 3 & 1 & 1 & 4 & 3 & 1 & 90.00 \\
\hline & 4 & 3 & 9 & 7 & 0 & 0 & 84.21 \\
\hline & 5 & 0 & 1 & 8 & 1 & 0 & 100.00 \\
\hline & 6 & 0 & 2 & 1 & 0 & 0 & 100.00 \\
\hline & 7 & 0 & 0 & 0 & 0 & 0 & 0.00 \\
\hline \multirow{7}{*}{$4 \mathrm{c} 3$} & 1 & 2 & 0 & 0 & 0 & 0 & 0.00 \\
\hline & 2 & 1 & 5 & 2 & 2 & 0 & 90.00 \\
\hline & 3 & 2 & 1 & 0 & 0 & 0 & 33.33 \\
\hline & 4 & 0 & 0 & 0 & 0 & 0 & 0.00 \\
\hline & 5 & 2 & 5 & 5 & 5 & 0 & 88.24 \\
\hline & 6 & 2 & 3 & 8 & 1 & 0 & 85.71 \\
\hline & 7 & 1 & 2 & 1 & 0 & 0 & 75.00 \\
\hline
\end{tabular}

\section{Conclusion}

A new method of discriminating direct injection-production connections was proposed. By applying computational geometry algorithms and percolation theory, using geology, static and dynamic, monitoring and stimulation data and considering various factors, this method can calculate water flooding directions in every well group in a layer at every date in production history. Many application examples prove that the method is more cost-saving and efficiency than testing among wells methods and is more reliable than applied mathematical methods. The method can be widely used to discriminate direct injection-production connections layer by layer under all kinds of geology conditions. The discrimination results can provide foundations for injection-production layers adjustment, well pattern improvement, water shut-off and profile control, and eventually oil recovery enhancement.

\section{References}

[1] Shang Fu-hua, Zheng Wei. Study on inferring interwell connectivity of injection- production system based on decision tree. Application Research of Computers. 2013, 30(7) 
[2] Xie Li-dong, Yan Hong, Zhu Zhong-kuan. Application of Grey Correlation System for Oil and Gas Wells in Waterflooding. JOURNAL OF OIL AND GAS TECHNOLOGY. 2008, 30 (3):141-143

[3] Zhao Hui,Yao Jun,LIU Ai-min,Wang Wei. Reservoir inter well dynamic connectivity inversion based on injection and production data. Journal of China University of Petroleum. 2010, 34(6):91-94.

[4] Jin Zhiyong; Liu Qipcng; Han Dong et. Inter-well connectivity analysis based on nonlinear time series. Petroleum Geology and Recovery Efficiency. 2009, 16(1):75-81

[5] Zhu Guojin Wang Yi, Ji LanminLi Yan Xu Kun. Study and application of the method for calculating injection production performance connected relationship. China Offshore Oil and Gas. 2011, 23(2)

[6] Chen Fuzhen, Jiang Hanqiao, YIN Fanghao, MENG Liang . EVALUATING METHOD OF THE INJECTOR-PRODUCER CONNECTIVITY BY KALMAN FILTERING. Petroleum Geology \& Oilfield Development in Daqing. 2012, 31(4)

[7] Mark de Berg, Otfried Cheong, Marc van Kreveld, Mark Overmars. Computational Geometry: Algorithms and Applications. Springer. 2007.

[8] Reinhard Diestel. Graph Theory. HIGHER EDUCATION PRESS. 2013.

[9] Ge Jia-li. The Modern Mechanics of Fluids Flow in Oil Reservoir, Petroleum Industry Press, 2003

[10]Chen Yuan-qian, Li Dang. Modern Petroleum Reservoir Engineering, Petroleum Industry Press, 2001

[11]Wang Shu-xi, Wu Zheng-xue. Improved Dijkstra Shortest Path Algorithm and its Application. Computer Science. 2012, 39(5): 223-228.

[12]LI Yan-yan. The Optimization Algorithm Dijkstra Implementation of Shortestpath Analysis. Geomatics \& Spatial Information Technology. 2014, 37(5): 172-173,190.

[13] Sun Yong, Liu Jing-xu, Song Liu-yong. Study of shortest paths optimization algorithm based on obstructed distance under complexity geographical environment. Science of Surveying and Mapping. 2014, 39(2): 37-41, 68.

[14][8] Li Zhong-wen, QIN Zhi-dong, WANG Quan-yu, NI Zhong-yu. Optimized genetic algorithm for shortest-path problem in game engine. Application Research of Computers. 2014, 31(1): 76-79.

[15] Tao Yu, Wu Zhong. Application of Optimized Ant Colony Algorithm in the Shortest Route Guidance. Journal of East China Jiaotong University. 2013, 30(6): 30-34,.

[16]Liu Yongqiang, Chang Qing, Xiong Huagang. Improved ant colony algorithm for shortest path problem in time-dependent networks. Journal of Beijing University of Aeronautics and Astronautics. 2009, 10: 1245-1248

[17]Franco P. Preparata, Michael lan Shamos. Computational Geometry: An Introduction. Springer. 1993.

[18]Wang Jiayie, Wang Wenping, Tu Changhe, Yang Chenglei. Computational Geometry and Its Applications. Science Press. 2011.

[19]Zhou Peide. Computational Geometry: Algorithms Analysis and Design. TSINGHUA UNIVERSITY PRESS. 2011. 